\title{
Extension of a Bernoulli Demand Inventory Model
}

\author{
Pritibhushan Sinha \\ 6 A J C Bose Road; Thakurpukur, Kolkata - 700063, \\ West Bengal, India \\ E-mail: p_sinha@indiatimes.com
}

\begin{abstract}
In this article a single-item, continuous monitoring $(s, Q)$ inventory model with probabilistic demand following Bernoulli distribution and probabilistic lead time of order replenishment is considered. The model extends a Bernoulli demand inventory model, available in the literature. We allow a non-zero reordering inventory position. Some properties are shown for the model and a search method is given to find the optimal values of the policy parameters. A numerical experiment is done for the model and some observations are made.
\end{abstract}

Keywords: : inventory, probabilistic, Bernoulli demand

\section{Introduction}

Inventory planning and control for materials is an important function in many types of businesses. Quite often probabilistic inventory models, rather than deterministic models, are appropriate, considering the uncertainty in demand and supply of the materials. As such, many types of this kind inventory models have been discussed in the literature. We may find these in Hadley and Whitin (1962), Heyman and Sobel (1990), Silver et al. (1998), to name only a few.

A type probabilistic inventory models is that in which the demand and/or the supply is modeled as a process that follows a Bernoulli distribution. Generalization of this is where such parameters may be seen as a compound Bernoulli process, a discrete distribution with probability mass concentrated on more than two points, etc. Dunsmuir and Snyder (1989) and Janssen et al. (1998) discussed periodic review, probabilistic inventory models with demand following compound Bernoulli process. They considered a model of $(R, s, Q)$ type. (The notation is as, $s$ : reordering inventory position, i.e., stock in hand + quantity of pending orders - back-logged demands; an order is placed whenever inventory position is $s$ or smaller; $Q$ : order quantity; an order of this constant quantity is placed in every occasion of ordering. $R$ - review period.) Approximate solutions for system characteristics as service level and average physical stock had been derived. Gullu et al. (1999) presented a finite horizon inventory model with known demands but uncertain supply. Supply is modeled using a three-point probability mass function. The supply is either completely available, partially available or not at all available. A special case considered was in which supply follows a Bernoulli process, such that, supply is either completely available or unavailable. Formulas for ordering quantities were given. This author discussed (Sinha 2008) a continuous review model with Bernoulli demand, and general, integer-valued probabilistic lead time, where order is placed only after inventory position reached zero $(s=0)$. Objective function was long term cost per unit time. Exactly optimal, closed-form solution was given for $Q$. The assumption of Bernoulli demand pattern may 
hold for slow-moving items in the context of industrial organizations or retail businesses. Placing order only when stock becomes zero would be appropriate if lead time is small enough and such conditions. But, the same model allowing $s \geq 0$ would broaden the scope of the same, as higher lead time and higher cost of lost sales may also be considered. In the present study, we attempt to do this. In particular, we have derived an expression for the same type of objective function for the model and have given a search method to determine an optimum solution.

Rest of this article is organized in the following way. In the next section, assumptions of the model are described in details. This is succeeded by analysis and optimization of the model. This section also includes numerical examples highlighting application of the model and some relevant observations. We end with some concluding remarks.

\section{Model}

\subsection{Notation}

The following notation is used in the subsequent analysis.

$p$ : Probability of demand being one unit during a unit of time;

$s$ : Reordering inventory position, $s=0,1, \ldots$;

$Q$ : Order quantity, $Q=s+1, s+2, \ldots$;

$L_{\mathrm{av}}$ : Average lead time;

$c$ : Cost of shortage per unit;

$h$ : Inventory holding cost per unit, per unit time;

$A_{0}$ : Ordering cost for an order;

$r$ : Profit per unit sold (over manufacturing and other such proportional costs);

$X_{i}$ : Random variable denoting demand during the $i$-th time unit, in a cycle. $X_{i}=1$ or 0 ;

$Y$ : Random variable representing lead time of an order. $Y=0,1, \ldots$;

C : Random variable denoting total cost in a cycle;

$T$ : Random variable denoting time length of a cycle.

\subsection{Assumptions in the Model}

We make the following assumptions to specify the model.

i) The system starts at time $=0$ with an inventory of $s$ units, when an order of replenishment of $Q(>s)$ units is placed.

ii) Demand for the item in every unit of time interval follows a Bernoulli distribution with parameter $p$. That is, $\boldsymbol{X}_{i}=1$ with probability $p(0<p \leq 1)$ and $\boldsymbol{X}_{i}=0$ with probability $(1-$ $p)$. The demand occurs at the end of the interval.

iii) Demands at different unit time intervals are mutually independent.

iv) As the inventory position again becomes $s$, an order of $Q$ units is placed and is received after $\boldsymbol{Y}$ time units, the lead time. $\boldsymbol{Y}$ follows an integer-valued discrete distribution. Minimum value of $Y$ is $L_{\min }(\geq 0)$ and maximum value is $L_{\max }(<\propto)$. Lead times are mutually independent and independent of demands.

v) Demand during a lead time, including the demand, if any, in the last unit time interval in a lead time, is unsatisfied and cost of shortage is $c$ /unit of shortage $(c \geq 0)$. Such shortage cost is a measure of goodwill loss, customer dissatisfaction etc.

vi) Inventory holding cost $h$, ordering $\operatorname{cost} A_{0^{\prime}}$ profit per unit sold $r$ are non-negative $\left(h, A_{0^{\prime}}\right.$ $r \geq 0$ ).

vii) Objective function considered is long term cost per unit time.

In the model, since demand during a lead time of supply is lost and $Q \geq s+1$, there would be at most one pending order at any time. There would be no cross-over of orders. At the time of order, inventory position and inventory in hand, i.e., physical stock, both would be $s$.

\section{Analysis and Optimization}

\subsection{Analysis of the Model}

The time in between successive two instants when inventory position becomes $s$ generates a renewal process, having identical and independent 
renewal cycles. The discrete random variable (r.v.) $C$ denotes the total cost, considering profits from sold units, costs of inventory holding, ordering and shortage in a cycle and the discrete r.v. $T$ denotes the time of a cycle. With the "Renewal Reward Theorem" (see, for example, Ross 1970), long term cost per unit time is $\mathrm{E}[\mathrm{C}] / \mathrm{E}[\mathrm{T}]$. We calculate the averages as described next.

\section{(a) Expected Inventory Cost in a Cycle}

Let $V\left(L, s_{1}\right)$ denote the average inventory cost for the situations when one order is placed at inventory position of $s_{1}$, lead time is $L$ and the next order is placed at $s$. We need to find $V(L, s)$. We may note that,

$V\left(L, s_{1}\right)=h s_{1}+p V\left(L-1, s_{1}-1\right)+(1-p) V\left(L-1, s_{1}\right), L>0, s_{1}>0$;

And,

$V(L, 0)=\frac{h}{2 p}(Q-s)(Q+s+1), L \geqslant 0$

$V\left(0, s_{1}\right)=\frac{h}{2 p}\left(Q+s_{1}-s\right)\left(Q-s_{1}+s+1\right), s_{1}>0$.

The term $V(L, s)$ can be calculated conveniently with the above recursive relations. We need to calculate, at most, $(L+1)(L+2) / 2$ terms, as given by the above equations, to calculate $V(L, s)$. Expected inventory holding cost in a cycle is given as,

$A_{1}(s, Q)=\sum_{L=L_{\min }}^{L_{\max }} V(L, s) \operatorname{Pr}\{Y=L\}$

It can be seen that,

$A_{1}(s, Q+1)=A_{1}(s, Q)+h(Q+1) / p$

\section{(b) Expected Shortage Cost in a Cycle}

It is given as,

$$
A_{2}(s)=c \sum_{L=s+1}^{L_{\max }} \sum_{k=s+1}^{L}(k-s){ }^{L} C_{k}(1-p)^{L-k} p^{k} \operatorname{Pr}\{\boldsymbol{Y}=L\}
$$

$A_{2}$ is independent of $Q$. The term ${ }^{L} C_{k}(1-p)^{L-k} p^{k}$ may also be calculated recursively (as in, Bowerman and Scheuer 1990).

\section{(c) Expected Time Length of a Cycle}

It is obtained as,

$B_{0}=\sum_{L=L_{\min }}^{L_{\max }}\left[L+\frac{Q}{p}-\frac{1}{p} \sum_{k=1}^{\min (L, s)} k{ }^{L} C_{k}(1-p)^{L-k} p^{k}\right.$

$\left.\frac{s}{p} \sum_{k=\min (L, s)+1}^{L}{ }^{L} C_{k} p^{k}(1-p)^{L-k}\right] \operatorname{Pr}\{Y=L\}$

with the convention that, in a summation a term is not considered if lower limit is higher than the upper limit. The term in [ ] is $Q / p$, for $L \leq s$, and $(Q-s) / p \leq$ $B_{0} \leq Q / p$. We also get,

$B_{0}(s, Q+1)=B_{0}(s, Q)+1 / p$.

With the terms as above, long term cost per unit time can be written as,

$K(s, Q)=\left(-r Q+A_{0}+A_{1}(s, Q)+A_{2}(s)\right) / B_{0}(s, Q)$

We may obtain optimal policy parameters $\left(s^{*}, Q^{*}\right)$ by a search method. Such a method would be faster with the use of the following observations. We further denote with $q(s)$ as the optimal order quantity when reordering inventory position is $s$.

Proposition 3.1: For the inventory model considered,

(i) $\mathrm{s}^{*} \leq \mathrm{L}_{\max }$.

(ii) $\mathrm{q}(\mathrm{s})$ is minimum $\mathrm{Q}$, such that the following holds,

$$
\frac{-r Q+A_{0}+A_{1}(s, Q)+A_{2}(s)}{B_{0}(s, Q)}<-(r / p)+h(Q+1)
$$

Proof:

(i) For any solution with $s>L_{\max ^{\prime}}$ we would have another solution with objective function value less or equal, decreasing reordering inventory position to $L_{\max }$ and increasing ordering quantity by the same amount.

(ii)

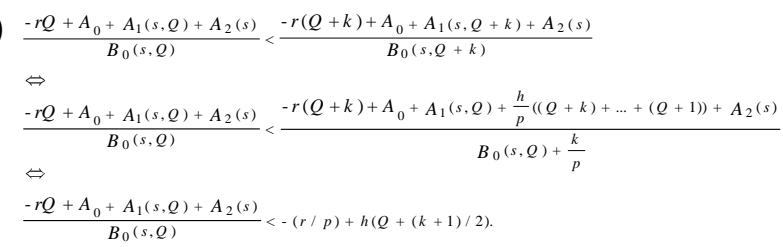

The above holds for $\forall k=1,2, \ldots$, if it holds for $k=1$. Thus, the statement is established.

Using the results as given in the proposition, we may get optimal solutions, $\left(s^{*}, Q^{*}\right)$, with the following method. 


\section{Method}

i. Calculate for $s=0,1, \ldots, L_{\max }$.

ii. For each $s$,

(a) Initially, $Q=s+1$. Calculate $A_{1}(s, s+1), A_{2}(s)$, $B_{0}(s, s+1)$. Calculate $K(s, s+1)$.

(b) Calculate for $K(s, Q)$ for $Q=s+2, s+3, \ldots$, till the condition (3.10) is satisfied, to get $q(s)$, the optimal ordering quantity $Q$, given reorder quantity is $s$. Calculate $A_{1}(s, Q), B_{0}(s$, $Q)$ recursively using (3.5) and (3.9).

iii. Values of $s$ and $q(s)$, giving minimum $K(s$, $Q)$, give $\left(s^{*}, Q^{*}\right)$.

\subsection{Numerical Experiment}

We consider the same numerical example as in Sinha (2008). The demand for an item of a particular brand in a retail store is 1 with probability 0.1 (or 0.2 ) and 0 with probability 0.9 , in an hour; and other conditions of the model hold. The average demand rate is $1 /$ day, assuming it operates 10 hours a day. Other data are taken as, $r=10 /$ unit, $A=100, h=0.006 /$ unit/hour. Calculations are done for two values of shortage cost c, 5/unit and 10/unit (a suitable currency unit may be used). Optimal reorder quantity $\left(s^{*}\right)$, optimal order quantity $\left(Q^{*}\right)$, long term cost per unit time for the optimal solutions $\left(K\left(s^{*}, Q^{*}\right)\right)$ for different values of average lead time $L_{\mathrm{av}}$ are shown. We consider two types of lead time, constant lead time (Table 1), and uniformly distributed random lead time (Table 2). In the latter case, the distribution is assumed to vary in $\pm 20 \%$ of the average. The method has been implemented with a macro, written in Visual Basic in MS Excel. A Pentium 4 personal computer, with Windows XP Professional operating system and with $2800 \mathrm{MHz}$ processor and $256 \mathrm{Mb}$ RAM (random access memory) has been used. Maximum computation time requirement, that has occurred for uniformly distributed lead time with average of 70 hours, has been 4 minutes 30 seconds.

\section{Discussions}

We have studied a probabilistic inventory model with Bernoulli demand and general discrete integervalued random lead time, with finite supports. It extends a similar model discussed earlier. No closedform optimal solutions for the policy parameters are available; but, these may be found with a search procedure. The following observations, for the model analyzed, can be made from numerical experiments done:

(a) Although it depends on the values of various parameters, order quantity given by the formula $\sqrt{2 \bar{D} A_{0} / h}, \bar{D}=p$, average demand per unit time, would be a good approximation for most of realistic data.

(b) At the same time an appropriate value of reordering inventory position also needs to be determined.

Table 1. Optimal Solutions for Constant Lead Time

\begin{tabular}{|c|c|c|c|c|}
\hline Obs. No. & Lead Time (hr.) & $\begin{array}{c}\text { Optimal Reordering Inventory } \\
\text { Position (s) }\end{array}$ & Optimal Ordering Quantity (Q) & $\begin{array}{l}\text { Optimal Cost per Unit Time } \\
\text { (hr.) } K(s, Q)\end{array}$ \\
\hline \multicolumn{5}{|l|}{$p=0.1, c=10$} \\
\hline 1 & 70 & 12 & 58.00 & -0.646584 \\
\hline 2 & 30 & 7 & 58.00 & -0.649320 \\
\hline 3 & 20 & 6 & 58.00 & -0.649861 \\
\hline 4 & 10 & 4 & 58.00 & -0.650317 \\
\hline 5 & 5 & 3 & 58.00 & -0.650490 \\
\hline 6 & 0 & 0 & 58.00 & -0.650586 \\
\hline \multicolumn{5}{|l|}{$p=0.1, c=5$} \\
\hline 1 & 70 & 12 & 58.00 & -0.646882 \\
\hline 2 & 30 & 7 & 58.00 & -0.649409 \\
\hline 3 & 20 & 6 & 58.00 & -0.649886 \\
\hline 4 & 10 & 4 & 58.00 & -0.650332 \\
\hline 5 & 5 & 3 & 58.00 & -0.650494 \\
\hline
\end{tabular}


Table 2. Optimal Solutions for Uniformly Distributed Random Lead Time

\begin{tabular}{|c|c|c|c|c|}
\hline Obs. No. & $\begin{array}{l}\text { Average Lead } \\
\text { Time (hr.) }\end{array}$ & $\begin{array}{c}\text { Optimal Reordering Inventory } \\
\text { Position }\left(\boldsymbol{s}^{*}\right)\end{array}$ & Optimal Ordering Quantity ( $Q$ ) & $\begin{array}{c}\text { Optimal Cost per Unit Time } \\
\text { (hr.) } \boldsymbol{K}(\boldsymbol{s}, \boldsymbol{Q})\end{array}$ \\
\hline \multicolumn{5}{|l|}{$p=0.1, c=10$} \\
\hline 1 & 70 & 13 & 58.00 & -0.646305 \\
\hline 2 & 30 & 7 & 58.00 & -0.649247 \\
\hline 3 & 20 & 6 & 58.00 & -0.649842 \\
\hline 4 & 10 & 4 & 58.00 & -0.650306 \\
\hline 5 & 5 & 3 & 58.00 & -0.650486 \\
\hline \multicolumn{5}{|l|}{$p=0.1, c=5$} \\
\hline 1 & 70 & 12 & 58.00 & -0.646647 \\
\hline 2 & 30 & 7 & 58.00 & -0.649362 \\
\hline 3 & 20 & 6 & 58.00 & -0.649874 \\
\hline 4 & 10 & 4 & 58.00 & -0.650325 \\
\hline 5 & 5 & 3 & 58.00 & -0.650491 \\
\hline \multicolumn{5}{|l|}{$p=0.2, c=5$} \\
\hline 1 & 70 & 21 & 83.00 & -1.498968 \\
\hline 2 & 30 & 12 & 82.00 & -1.504598 \\
\hline 3 & 20 & 9 & 82.00 & -1.505712 \\
\hline 4 & 10 & 6 & 82.00 & -1.506585 \\
\hline 5 & 5 & 4 & 82.00 & -1.506918 \\
\hline
\end{tabular}

(c) Higher lead time, or uncertainty in lead time, decreases profit rate and should be tried to be avoided. But the effect of such conditions can be decreased to a large extent by having a suitable amount of safety stock, through the reordering inventory position.

The assumption of Bernoulli demand pattern in the model narrows the scope of application of the same. Yet it may be a suitable in some practical situations. In such cases, the present model would be of use to inventory planners.

\section{Acknowledgement}

The author very much thanks the Editor-inChief and the reviewer for their help and the suggestions to improve the article.

\section{References}

Bowerman, P. N. and Scheuer, E. M. (1990). Calculation of the Binomial Survivor Function. IEEE Transactions on Reliability 39 (2, June), pp. 162-166.

Dunsmuir, W.T.M. and R.D. Snyder, R.D. (1989). Control of inventories with intermittent demand. European Journal of Operational Research 40(1), pp. 16-21.

Gullu, R., Onol, R. and Erkip, N. (1999). Analysis of an inventory system under supply uncertainty. International Journal of Production Economics 59 (1-3, March), pp. 377385.

Hadley, G. and Whitin, T. (1963). Analysis of Inventory Systems. Prentice-Hall, Englewood Cliffs, NJ.

Heyman, D. P. and Sobel, M. J. (Eds.). (1990). Handbooks in OR \& MS, Vol. 2 (Stochastic Models). Elsevier (NorthHolland), Amsterdam.

Janssen, F., R., Heuts, R. and de Kok, T. (1998). On the (R, $s$, $Q)$ model when demand is modeled as a compound Bernoulli process. European Journal of Operational Research 104(3), pp. 423-436.

Ross, S.M. (1990). Applied probability Models with Optimization Applications. Holden-Day, San Francisco.

Silver, E.A., Pyke, D.F. and Peterson, R. (1998). Inventory Management and Production Planning and Scheduling. Wiley, New York.

Sinha, P. (2008). A Note on a Bernoulli Demand Inventory Model. Decision 35(1), pp. 19-30. 
Pritibhushan Sinha is a freelance consultant in Operations management and Market Research. He has served in industrial and academic organizations for substantial amount of time. In research, he is interested in applied models of operations research / management and practicable solution methods and has published in the journals such as, Annals of Operations Research, International Transactions in Operational Research, Opsearch, International Journal of Applied Management Science, Decision, International Journal of Market Research. Mr. Sinha graduated from the Indian Institute of Technology Kharagpur in the branch of Manufacturing Science and Engineering (1987). Later, he pursued doctoral studies in the area of Operations Research \& Systems Analysis in the Indian Institute of Management Calcutta and obtained the title, "Fellow of the Indian Institute of Management Calcutta" (1994). 